Lavega Burgués, Pere; March Llanes, Jaume; Filella Guiu, Gemma (2013). Juegos deportivos y emocionès. Propiedades psicométricas de la escala GES para ser aplicada en la Educación Física y el Deporte. Revista de Investigación Educativa, 31 (1), 151-165. http://dx.doi.org/10.6018/rie.31.1.147821

\title{
JUEGOS DEPORTIVOSY EMOCIONES. PROPIEDADES PSICOMÉTRICAS DE LA ESCALA GES PARA SER APLICADA EN LA EDUCACIÓN FÍSICAY EL DEPORTE
}

\author{
Pere Lavega $a^{a}$; Jaume March $^{b}$ Gemma Filella ${ }^{b}$, \\ a Instituto Nacional de Educación Física de Cataluña (INEFC), Universidad de Lleida \\ Pere Lavega: plavega@inefc.udl.cat

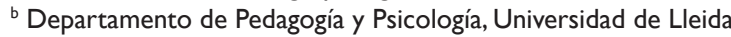

\section{RESUMEN}

El objetivo de este estudio fue validar la escala juegos deportivos y emociones (GES), basada en tres tipos de emociones: positivas, negativas y ambiguas, en las 4 situaciones de juegos deportivos correspondientes a diferentes dominios de acción motriz. El trabajo se realizó con 851 estudiantes universitarios en ciencias de la actividad física y del deporte de 4 universidades españolas, 1 inglesa y 1 portuguesa.

El GES presentó una correcta fiabilidad mostrando valores adecuados de consistencia interna. Se obtuvo un buena validez de constructo que confirmó la estructura factorial del cuestionario en los 3 tipos de emociones y demostró la necesidad de diferenciar entre los 4 dominios de acción motriz. La validez convergente mostró buenos resultados al comparar el GES y el cuestionario Profile of moods states (POMS). La validez de contenido confirmó que la estructura del GES es adecuada para estudiar la relación entre juegos deportivos y emociones. En conclusión, el cuestionario ha demostrado su validez y fiabilidad para registrar la intensidad de las emociones en la práctica de juegos deportivos.

Palabras clave: educación física; juegos; emociones; dominios de acción motriz.

\footnotetext{
Correspondencia:

Pere Lavega i Burgues: Facultad Ciencias de la Educación. Departamento de Pedagogía y Psicología. Universidad de Lleida. Avda. Estudio General, 4. 25001 Lleida.

Jaume March: jaume.march@cmb.udl.cat

Gemma Filella Guiu: Universitat de Lleida. Facultat de Ciències de l'Educació. Av. de l'Estudi General, 4. 25001 Lleida. Tel. 973706572/6501. gfilella@pip.udl.cat
} 


\title{
SPORTS GAMES AND EMOTIONS. PSYCHOMETRIC PROPERTIES OF THE GAMES AND EMOTIONS SCALE (GES) AND ITS USE IN PHYSICAL EDUCATION
}

\begin{abstract}
The aim of this study was to validate the Games and Emotions Scale (GES) based on three types of emotions (positive, negative, and ambiguous) in four sports game scenarios related to different motor action domains. The study was conducted on 851 Physical Education and Sports students from four Spanish, one English, and one Portuguese university.

The GES yield an adequate reliability and values of internal consistency. We obtained good construct validity that confirmed the factor structure of the scale in the three types of emotions and also highlighted the need to differentiate between the four motor action domains.

Convergent validity showed good results when comparing the GES with the Profile of moods states questionnaire (POMS). Content validity confirmed that the structure of the GES is adequate to study the relation between sports games and emotions. In conclusion, validity and reliability of this questionnaire to record the intensity of emotions in sports games were confirmed.
\end{abstract}

Keywords: Physical Education; games; emotions; motor action domains.

\section{INTRODUCCIÓN}

Cualquier juego deportivo constituye un auténtico laboratorio de reacciones emocionales (Parlebas, 2001; Lagardera, 1999), que hace que cada alumno o deportista viva de manera distinta una sesión de educación física, un entrenamiento o un partido de campeonato. La comprensión de la relación existente entre los distintos tipos de juegos deportivos y las distintas clases de emociones es un tema de máxima importancia para el diseño, la aplicación y la evaluación de programas en el campo de la actividad física y el deporte (AFD) orientados hacia el bienestar personal y social.

Entre los principales instrumentos que se han empleado para valorar emociones en la AFD, destacamos el Befindlichkeitsskalen (BFL: Alfermann, 2003) que considera la activación y la evaluación de las emociones, el Multiple Affect Adjective Check List (MAACL: Zuckerman y Lubin, 1965), el Profile Mood States (POMS: McNair, Lorr y Droppleman,1971), el Positive Affect Negative Affect Schedule (PANAS: Watson, Clark, y Tellegen,1988), el Exercise Induced Feeling Inventory (EFI: Gauvin y Rejeski, 1993), la Subjective Exercise Experiences Scale (SEES: McAuley y Courneya, 1994), y la Brunel Mood Scale (BRUMS: Terry, Lane, Lane y Keohane, 1999).

La mayoría de instrumentos sigue la estructura definida por el POMS y el PANAS (Terry, Lane y Fogarty, 2003). Los estudios realizados para determinar el perfil de estados de ánimo del POMS y para generar escalas derivadas del mismo (Grove y Prapavessis, 1992; Terry, Lane, Lane, y Keohane, 1999; Terry, Lane, y Fogarty, 2003) consideran seis estados de ánimo: ira, confusión, depresión, fatiga, tensión y vigor. Estas escalas se han empleado para predecir el nivel de rendimiento en el deporte (e.g., Beedie, Terry y Lane, 2000; Morgan, Brown, Raglin, O'Connor y Ellickson, 1987). El 
PANAS, en cambio, considera tres estados de afecto positivos (felicidad, la atención, la tranquilidad) y cinco de negativos (tristeza, culpabilidad, miedo, fatiga, timidez).

Se observa un limitación común en estas escalas ya que no fueron diseñadas para su aplicación en el campo de la AFD (Hanin, 2000). El POMS se desarrolló para ser usado con población clínica, y este motivo explica en gran medida, el predominio de estados de ánimo negativos (cinco) respecto a los positivos (uno). Aunque el BRUMS fue una adaptación concebida para la AFD sigue las mismas directrices que el POMS. El PANAS se diseñó para estudiar estados afectivos en la vida diaria, observando que cuando la persona siente fatiga, miedo o timidez tiende a tener un estado de afecto negativo; sin embargo cuando se siente atención, tranquilidad o felicidad se tiene un estado de afecto positivo.

No obstante en ambos grupos de cuestionarios se confunde la emoción con estados de ánimo (Ekman y Davidson, 1994). Por ejemplo a veces se introducen adjetivos como cansado, inmóvil o activo que responden a una perspectiva energética, en otras ocasiones se habla de confuso, desconcertado o atento a partir de una visión más conductual que emocional. De hecho, la emoción es un estado complejo del organismo caracterizado por una excitación o perturbación que predispone a una respuesta organizada (Bisquera, 2003), mientras que el estado de ánimo se refiere a un estado emocional de mayor duración y menor intensidad (Parkinson, Totterdell, Briner y Reynols, 1996), y las emociones tienen una duración breve y provocadas por un estímulo específico (Lane y Terry, 2000).

Entre los instrumentos relacionados, merece una atención distinta The Emotion Questionnaire (SEQ: Jones, Lane, Bray, Uphill, y Catlin, 2005) que recoge cinco subescalas de emociones, aunque sigue sin atender la distinción de los tres grupos de emociones (positivas, negativas y ambiguas) que consideran Lazarus $(1991,2000)$ y Bisquerra (2000, 2003), para nosotros autores de referencia en el marco teórico de las emociones desde el que se sustentan nuestras hipótesis.

Paralelamente, existen instrumentos específicos para una emoción o grupo de emociones, destacando: Bredemeier Athletic Aggression Inventory (BAAGI: Bredemeier, 1975); Competitive State Anxiety Inventory (CSAI-2: Martens, Burton, Vealey, Bump, y Smith, 1990); Sport Anxiety Scale (SAS: Smith, Smoll y Schultz, 1990); Aggressive Tendencies in Basketball Questionnaire (ATBQ: Duda, Olson, y Templin, 1991); Physical Activity Enjoyment Scale (PACES: Kendzierski y DeCarlo, 1991); Flow State Scale (FFSS: Jackson y Marsh, 1996; García Calvo, Jiménez, Santos-Rsoa, Rena y Cervelló, 2008); y Trait Flow Scale (TFS: Jackson, Kimiecik, Ford, y Marsh, 1998). Sin embargo, una vez más la presencia de cuestionarios no específicos para la AFD y su concreción en una o varias emociones impedía adoptar un estudio multidimensional sobre las emociones que se originan en los juegos deportivos.

La revisión exhaustiva de las escalas constató que ningún instrumento diferenciaba los tres grupos de emociones: positivas, negativas y neutras establecidas por los autores de referencia (Lazarus, 1999, 2000; Bisquerra, 2000). Tampoco se observó que tuviesen en cuenta la influencia de los dominios de acción motriz (Parlebas, 2001) que, atendiendo al criterio de interacción motriz, corresponden a las categorías de psicomotor, cooperación, oposición y cooperación-oposición.

Por este motivo, en 2007 se inició un proceso de investigación interdisciplinario entre el grupo de investigación en juegos deportivos (GREJE), del INEFC-Universidad de 
Lleida, integrado por investigadores de diferentes centros de Europa y Latinoamérica y el grupo de investigación en orientación psicopedagógica especializado en la educación de competencias emocionales (GROP) de la Universidad de Lleida y Barcelona. Este trabajo cooperativo permitió elaborar el cuestionario GES que se presenta y valida en este artículo.

Cualquier profesional de la motricidad (profesor de educación física, entrenador o animador) debe decidir constantemente que tipo de situaciones motrices presentar a sus destinatarios (alumnado, deportistas o turistas) para conseguir los efectos esperados (Parlebas, 2001) para su proyecto de intervención (pedagógico, deportivo o recreativo). El juego motor o deportivo es una manifestación muy utilizada en los diferentes ámbitos de intervención, aunque a menudo se presenta de modo parcelado, como si se tratara de una pieza más del mosaico de técnicas independientes, empleadas sin apenas interrelaciones. Es necesario recordar que el profesorado debe conocer los procedimientos para que el alumnado aprendan mejor, $\mathrm{y}$, por supuesto, ha de ser consciente de los objetivos propuestos y de los criterios e instrumentos de evaluación que va a utilizar (De Moya, Hernández, Hernández y Cózar, 2011; Iglesias, 2009). Por ese motivo resulta imprescindible partir de una sólida conceptualización teórica y científica que permita establecer una unidad para todos los contextos de intervención motriz (Parlebas, 1968, 2010; During, 1981, Lagardera, 1999).

La praxiología motriz permite sistematizar los distintos tipos de prácticas motrices o juegos a partir de la noción de dominio de acción motriz, referido al "campo en el que las prácticas motrices son consideradas homogéneas respecto a criterios pertinentes y precisos de acción motriz" (Parlebas, 2001, p. 161).

Al aplicar el criterio del tipo de interacción motriz que mantienen los protagonistas de cualquier juego, se establecen cuatro dominios de acción motriz: a) Juegos Psicomotores en los que no existe interacción motriz entre los participantes; b) Juegos de Cooperación, en los que dos o más participantes interactúan ayudándose para resolver un objetivo común; c) Juegos de Oposición en que cada jugador se enfrenta a uno o más adversarios; y d) Juegos de Cooperación-oposición en los que los jugadores de un mismo equipo cooperan entre ellos para vencer a otros rivales generalmente también organizados en equipo.

Cada uno de estos cuatro dominios de acción motriz se puede realizar con o sin competición, o sea, con o sin victoria final. Cuando aparece la competición los jugadores quedan clasificados en ganadores y perdedores, cosa que no sucede cuando los juegos no tienen un final establecido.

En cuanto al marco teórico de las emociones se constata una gran diversidad de definiciones y clasificaciones (Kleinginna y Kleinginna, 1981), y una vez hecha la revisión, se ha optado por la clasificación de las emociones propuesta por Lazarus (1991, 2000) y (Bisquerra, 2000), que considera tres tipos de emociones: emociones positivas (alegría, humor, amor y felicidad), las emociones negativas (miedo, ansiedad, ira, tristeza, rechazo, vergüenza) y las emociones neutras o ambiguas: sorpresa, esperanza y compasión.

A continuación se presenta el tema central de cada emoción en función del daño o beneficio que se obtiene durante cada juego (Bisquerra, 2000). 
- Alegría: Disfrutar de un suceso durante el juego satisfactorio.

- Humor: Inspira alegría y mueve a la risa durante el juego.

- Amor: Desear o participar en afecto con otra persona del juego, habitualmente pero no necesariamente recíproco.

- Felicidad: Hacer progresos razonables hacia el logro del objetivo que plantea el juego.

- Miedo: Sentir peligro físico real e inminente, concreto y arrollador durante el juego.

- Ansiedad: Enfrentarse a una amenaza incierta durante el juego.

- Ira: Sentir una ofensa contra mi o mi equipo durante el juego.

- Tristeza: Experimentar una pérdida irreparable durante el transcurso del juego.

- Rechazo: No admitir o aceptar a otra persona que participa en el mismo juego.

- Vergüenza: Fracasar en vivir de acuerdo con el yo ideal. Timidez que se siente ante determinadas situaciones de juego que impide o inhibe la realización de una acción motriz.

- Sorpresa: Reacción a alguna situación de juego imprevista.

- Esperanza: Confiar en qué ocurrirá o se logrará el objetivo de juego que se desea alcanzar.

- Compasión: Sentirse afectado por el sufrimiento de otro participante en el juego y sentir el deseo de querer ayudarle.

El objetivo de esta investigación ha sido estudiar en participantes universitarios las propiedades psicométricas del GES (Games and Emotions Scale) y su capacidad discriminativa entre los cuatro dominios de acción motriz, mediante juegos con y sin victoria. Para ello se planteó estudiar la fiabilidad, la validez de constructo la validez convergente y la validez de contenido.

\section{MÉTODO}

\section{Participantes}

La escala GES se aplicó a 851 estudiantes universitarios en ciencias de la actividad física y del deporte de las universidades de Lleida, Barcelona, Vitoria, Católica de Murcia, Gales -en su sede en Málaga-, todos de primer curso que participaron voluntariamente en un programa de educación física emocional: Hombres, $n=318(37,37 \%)$ $\left(M_{\text {edad }}=20,15, S D=3,28\right)$ y mujeres, $n=533(62,63 \%)\left(M_{\text {edad }}=20,13, S D=3,61\right)$. El rango de edad fue de 18- 44 años.

\section{Diseño}

Se diseñó un estudio cuasi-experimental en el que los sujetos contestaron el cuestionario GES tras realizar cada uno de los ocho juegos (8 momentos) distribuidos en dos sesiones. El cuestionario POMS se cumplimentó en 4 momentos distintos (al inicio y fin de cada sesión), con el objetivo de llevar a cabo el estudio de las propiedades psicométricas del cuestionario GES. 


\section{Instrumentos}

La escala GES recoge las puntuaciones de 0 a 10 de los sujetos, correspondientes a las 13 emociones (positivas, negativas y ambiguas), después de realizar diferentes juegos deportivos. Concretamente, se utilizaron 8 juegos, la mitad con posibilidad de victoria y la otra mitad sin. Los juegos pertenecían a los 4 dominios de acción motriz: psicomotor (los jugadores intervienen sin interactuar motrizmente con otros participantes), cooperación (los jugadores interactúan colaborando con sus compañeros), oposición (los jugadores interactúan oponiéndose a sus adversarios) y cooperación-oposición (los jugadores interactúan colaborando con sus compañeros de equipo y oponiéndose a los rivales del equipo contrario). De cada dominio se realizó un juego con victoria (proclamando un vencedor y un perdedor) y un juego sin victoria (sin competición). En el anexo 1 se puede observar la estructura del cuestionario.

Para analizar la validez convergente, se aplicó la versión reducida de la escala POMS (Balaguer, Fuentes, Meliá y García Merita, 1994) a una submuestra de 115 participantes. Este cuestionario constaba de 29 ítems asociados a seis estados de ánimo, y se aplicó al principio y final de cada sesión de juegos deportivos.

\section{Procedimiento}

Antes de realizar los juegos los participantes recibieron cuatro horas y treinta minutos de formación teórica y práctica en las emociones, con el objetivo de unificar criterios de respuesta y habilidades para identificar las 13 emociones estudiadas en esta investigación. Se realizaron dos sesiones de una hora y treinta minutos para explicar los principios teóricos básicos subyacentes de educación emocional: concepto, componentes, la clasificación y el significado de las emociones en el contexto de la educación física. En estas sesiones, los estudiantes aprendieron cómo identificar las trece emociones por medio de ejercicios de situaciones de juego. También se familiarizaron con el cuestionario GES. Esta formación fue impartida por un grupo de investigadores formados en educación emocional. El trabajo de recogida de información se llevó a cabo una vez se confirmó que los estudiantes no tenían más dudas en cuanto a la identificación de las emociones.

Tras confirmar que el alumnado podía realizar la identificación de las 13 emociones y emplear correctamente el GES se procedió a realizar dos sesiones prácticas de 90 minutos. Los participantes se distribuyeron en grupos de 40 estudiantes. Todo el profesorado empleó las mismas estrategias didácticas, realizando los juegos en las mismas condiciones. En cada sesión se realizó un juego representativo de cada uno de los dominios de acción motriz (uno con victoria y otro sin victoria).

Una vez finalizado cada juego deportivo, los participantes se dirigieron en silencio a una zona de la pista para cumplimentar el cuestionario GES. Acto seguido los participantes, dejaban su hoja de registro y participaban en el juego siguiente.

Al finalizar el último juego de esa sesión el alumnado entregó la hoja al profesor/a de esa sesión con el fin de evitar cualquier tipo de manipulación de los datos a posteriori. Además, si se hallaba un cuestionario con alguna tachadura o incorrección era desestimado para el tratamiento estadístico de los datos. 


\section{Análisis de datos}

En primer lugar se realizó un análisis descriptivo de los ítems de la escala GES. Acto seguido se estudió la validez de constructo a través de un análisis factorial confirmatorio. Posteriormente, se estudió la fiabilidad a través de la consistencia interna, calculando el alfa de Cronbach. La validez convergente se analizó comparando los resultados en los cuestionarios GES y POMS. Se compararon las emociones negativas del GES con los factores fatiga, depresión, tensión y hostilidad del POMS. Por otra parte, se compararon las emociones positivas del GES con el factor vigor del POMS. Las emociones ambiguas no se compararon con ningún factor del POMS debido a que no tienen ninguna correspondencia.

Por último, el estudio de la validez de contenido se llevó a cabo mediante la valoración de veinticuatro jueces, doce especialistas en emociones y doce en juegos deportivos. Para ello, se les entregó un pequeño dossier con una explicación del proceso en el que iban a participar así como una definición global de la escala y de los factores que se pretendían estudiar. Se utilizó el programa IBM SPSS 18.0 y AMOS 18.0 para llevar a cabo los distintos análisis estadísticos.

\section{RESULTADOS}

\section{Análisis descriptivos}

Globalmente, hay que destacar que las emociones positivas obtuvieron los valores de intensidad más elevados en los cuatro dominios de acción motriz y en los distintos resultados de la intervención (ganador, perdedor, sin victoria). Las emociones ambiguas

TABLA 1

ESTADÍSTICOS DESCRIPTIVOS DE LA INTENSIDAD DE LAS EMOCIONES, EN FUNCIÓN DEL DOMINIO DE ACCIÓN MOTRIZ, EL RESULTADO DE LA INTERVENCIÓN Y EL TIPO DE EMOCIÓN

\begin{tabular}{|c|c|c|c|c|c|c|c|c|c|c|}
\hline \multirow{3}{*}{$\begin{array}{c}\text { Dominio de acción } \\
\text { motriz }\end{array}$} & \multirow{3}{*}{$\begin{array}{l}\text { Tipo de } \\
\text { emoción }\end{array}$} & \multicolumn{6}{|c|}{ Resultado de la intervención } & \multirow{3}{*}{$\begin{array}{l}\text { Asime- } \\
\text { tría }\end{array}$} & \multirow{3}{*}{$\begin{array}{l}\text { Curto- } \\
\text { sis }\end{array}$} & \multirow{3}{*}{$\begin{array}{l}\text { Alfa de } \\
\text { Cronbach }\end{array}$} \\
\hline & & \multicolumn{2}{|c|}{ Ganador } & \multicolumn{2}{|c|}{ Perdedor } & \multicolumn{2}{|c|}{ Sin victoria } & & & \\
\hline & & Media & DT & Media & DT & Media & DT & & & \\
\hline \multirow{3}{*}{ Psicomotor } & positiva & 5,86 & 3,23 & 4,20 & 2,87 & 3,96 & 3,30 & 0,01 & $-1,25$ & 0,74 \\
\hline & negativa & 0,86 & 2,04 & 1,89 & 2,73 & 0,48 & 1,52 & 2,51 & 5,64 & 0,73 \\
\hline & ambigua & 3,29 & 3,45 & 2,92 & 3,10 & 1,82 & 2,75 & 0,95 & $-0,45$ & 0,72 \\
\hline \multirow{3}{*}{ Cooperación } & positiva & 7,45 & 2,80 & 4,78 & 3,02 & 6,40 & 3,12 & $-0,60$ & $-0,73$ & 0,78 \\
\hline & negativa & 0,86 & 2,03 & 2,18 & 2,92 & 0,29 & 1,06 & 2,44 & 5,19 & 0,75 \\
\hline & ambigua & 3,55 & 3,53 & 3,12 & 3,18 & 2,47 & 3,23 & 0,69 & $-0,94$ & 0,75 \\
\hline \multirow{3}{*}{ Oposición } & positiva & 6,87 & 2,97 & 4,74 & 3,03 & 5,83 & 3,03 & $-0,56$ & $-0,76$ & 0,76 \\
\hline & negativa & 0,70 & 1,65 & 2,03 & 2,73 & 0,99 & 1,95 & 1,85 & 2,51 & 0,79 \\
\hline & ambigua & 3,79 & 3,32 & 3,41 & 3,17 & 2,94 & 3,10 & 0,45 & $-1,14$ & 0,73 \\
\hline \multirow{3}{*}{$\begin{array}{l}\text { Cooperación- } \\
\text { Oposición }\end{array}$} & positiva & 5,99 & 3,45 & 5,45 & 3,13 & 5,39 & 2,89 & $-0,46$ & $-0,84$ & 0,79 \\
\hline & negativa & 0,95 & 2,03 & 1,94 & 2,75 & 1,25 & 2,10 & 1,67 & 1,87 & 0,77 \\
\hline & ambigua & 3,35 & 3,29 & 3,59 & 3,24 & 3,22 & 3,04 & 0,36 & $-1,20$ & 0,76 \\
\hline
\end{tabular}


registraron intensidades intermedias y las emociones negativas los valores más bajos en todos los casos (Tabla 1).

En cuanto a las emociones positivas, el dominio psicomotor registró los valores más bajos en los distintos resultados de la intervención, siendo el dominio de cooperación el que obtuvo los resultados más elevados. En relación a las emociones negativas solo cabe destacar que los valores más altos se observaron en el dominio Cooperación-oposición, ya que el resto de dominios se comportaron de forma muy parecida. Por último, en las emociones ambiguas, el dominio psicomotor obtuvo los valores más bajos y el dominio Cooperación-oposición los más elevados. En la tabla 1 se muestran las intensidades de estas emociones comentadas, en función del resultado de la intervención.

Los valores de asimetría y curtosis (tabla 1) cumplieron los criterios de normalidad propuestos por Curran,West y Finch (1996). En todos los casos la asimetría fue inferior a 2.0 y la curtosis inferior a 7.0.

\section{Validez de constructo}

Llevamos a cabo un análisis factorial confirmatorio (AFC) para analizar la estructura de 3 factores del cuestionario, en cada uno de los 4 dominios de acción motriz, ya que el AFC inicial sin diferenciar entre dominios de acción motriz proporcionaba una alta significación $(\mathrm{p}<, 001)$. Se utilizó el método de máxima-verosimilitud en todos los casos.

\section{Dominio psicomotor}

El AFC mostró los siguientes índices de ajuste: CMIN/g.l. = 7,014; NFI = ,813; $\mathrm{IFI}=$,836; CFI = ,833; RMSEA $=, 08$ (LO90 = ,072 - HI90 = ,09). Los pesos factoriales oscilaron entre el ,50 de la tristeza y el ,77 de la alegría. Todos los pesos fueron significativos $(\mathrm{p}<, 05)$ y superiores en la dimensión hipotetizada.

\section{Dominio de cooperación}

El AFC mostró los siguientes índices de ajuste: $\mathrm{CMIN} / \mathrm{g} .1 . \mathrm{l}=3,041 ; \mathrm{NFI}=$,926; IFI = ,949; CFI = ,948; RMSEA = ,049 (LO90 = ,041 - HI90 = ,057). Los pesos factoriales oscilaron entre el, 42 de la ira y el ,83 de la ansiedad. Todos los pesos fueron significativos $(p<.05)$ y superiores en la dimensión hipotetizada.

Dominio de oposición.

El AFC mostró los siguientes índices de ajuste: $\mathrm{CMIN} / \mathrm{g} .1 . \mathrm{l}=3,97$; NFI $=, 889$; IFI = ,915; CFI = ,914; RMSEA = ,059 (LO90 = ,051 - HI90 = ,067). Los pesos factoriales oscilaron entre el , 47 del miedo y el ,81 de la alegría. Todos los pesos fueron significativos $(p<, 05)$ y superiores en la dimensión hipotetizada.

Dominio de Cooperación-Oposición.

El AFC mostró los siguientes índices de ajuste: $\mathrm{CMIN} / \mathrm{g} .1$. = 3,6; NFI = ,906; IFI = ,93; $\mathrm{CFI}=, 929 ; \mathrm{RMSEA}=, 055$ (LO90 $=, 048-\mathrm{HI} 90=, 063)$. Los pesos factoriales oscilaron entre el ,44 de la compasión y el ,89 de la alegría. Todos los pesos fueron significativos $(p<, 05)$ y superiores en la dimensión hipotetizada.

En la figura 1, se describen los resultados del AFC en los cuatro dominios de acción motriz. 

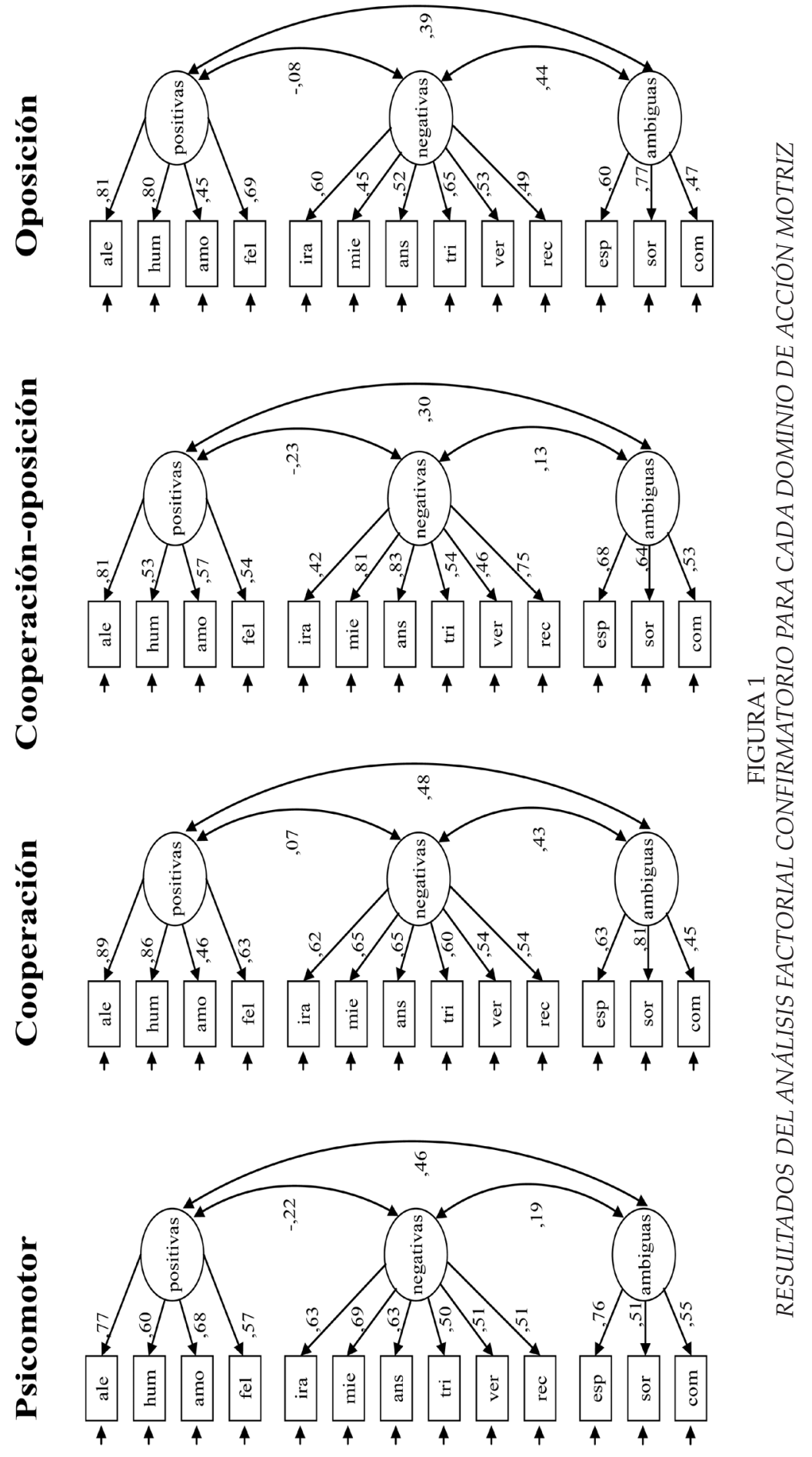


\section{Análisis de fiabilidad}

La consistencia interna medida a través del alfa de Cronbach de la escala GES fue de 0.88 , indicando una alta fiabilidad. En la tabla 1, se presentan los valores de alfa de Cronbach para cada dominio de acción motriz y en función de cada tipo de emoción, observándose igualmente unos niveles aceptables.

\section{Validez convergente}

Se analizó mediante el coeficiente de correlación de Pearson la relación entre las puntuaciones a los cuestionarios GES y POMS. Los resultados para cada factor del POMS fueron: Tensión-Ansiedad, se observó una correlación significativa $(\mathrm{r}=, 379$, $\mathrm{p}<, 01)$ con el valor promedio de las emociones negativas del GES. Depresión-Abatimiento, se observó una correlación significativa $(\mathrm{r}=, 383, \mathrm{p}<, 01)$ con el valor promedio de las emociones negativas del GES. Rabia-Hostilidad, se observó una correlación significativa $(\mathrm{r}=, 422, \mathrm{p}<, 01)$ con el valor promedio de las emociones negativas del GES. Fatiga-Inmovilidad, se observó una correlación no significativa $(r=, 268, p>, 01)$ con el valor promedio de las emociones negativas del GES. Vigor-Actividad, se observó una correlación significativa $(\mathrm{r}=, 227, \mathrm{p}<, 05)$ con el valor promedio de las emociones positivas del GES.

\section{Validez de Contenido}

Se estudió el grado en que el conjunto de los ítems del cuestionario representaba adecuadamente la relación entre los juegos deportivos y las emociones mediante la valoración de 24 jueces de prestigio internacional, 12 especialistas en juegos deportivos y 12 especialistas en educación emocional.

La labor de los expertos era valorar el cuestionario, de 0 (puntuación totalmente negativa) a 10 (puntuación totalmente positiva), mediante 6 preguntas, A continuación se presentan las medias observadas en cada aspecto analizado: a) Los juegos seleccionados son adecuados en relación con la fundamentación teórica de la que partimos $(\mathrm{M}=8,50, \mathrm{DT}=0,905)$; b) Las emociones escogidas son adecuadas para el cuestionario $(\mathrm{M}=8,58, \mathrm{DT}=1,084)$; c) El orden en el que se han enumerado las emociones es adecuado $(\mathrm{M}=8,75$, DT $=1,055)$; $\mathrm{d})$ Las expresiones utilizadas en el cuestionario son fácilmente comprensibles para los alumnos universitarios $(\mathrm{M}=8,42, \mathrm{DT}=1,084)$; e) La estructura del cuestionario es adecuada para la finalidad de la investigación $(\mathrm{M}=9,08, \mathrm{DT}=0,900)$; $\mathrm{f})$ La estructura del cuestionario facilita las respuestas. $(\mathrm{M}=9,17$, $\mathrm{DT}=0,577)$. A partir de estos datos se puede afirmar la unanimidad entre los jueces.

\section{DISCUSIÓN}

El objetivo del presente estudio fue analizar las propiedades psicométricas del GES en una muestra de participantes universitarios en ciencias de la actividad física y del deporte. Los resultados proporcionaron unos valores adecuados de fiabilidad y validez contando con unas propiedades psicométricas aceptables. 
En el análisis descriptivo se puso de manifiesto la influencia del tipo de emociones que en los posteriores análisis se detectaron. Aunque el funcionamiento de las tres emociones fue muy distinto, la escala de medida propuesta se adaptó suficientemente al no observarse defectos en asimetría y curtosis, lo que implicó una correcta aceptabilidad. A diferencia de otros estudios en situaciones de alta competición deportiva (e.g., Jones, Swain, y Hardy, 1993; Jones, y Sheffield, 2007; Hanin, 2000; Mullen, Lane y Hanton, 2009; y Uphill y Jones, 2007), en los que se registraron valores más intensos de emociones negativas, en nuestro caso los participantes fueron expuestos a juegos con un nivel de competición muy moderado.

Con respecto a la fiabilidad, los resultados mostraron que el GES presentó valores adecuados de consistencia interna.

Respecto a la validez de constructo del GES, los resultados revelaron dos aspectos. Primero, la necesidad de tener en cuenta los cuatro dominios de acción motriz. Esto coincide con otros estudios fundamentados en el mismo marco teórico de la praxiología motriz, que observaron que los dominios de acción motriz se comportan de manera distinta en el ámbito de transferencia de aprendizajes (Collard, Oboeuf, Almaidi, 2007; Parlebas y Dugas, 1998 ) o en efectos sobre la cohesión social (Delaunay, 1981; Oboeuf, Collard y Gerard, 2008).

Segundo, que en cada dominio de acción motriz, se observó el mismo patrón de comportamiento de las emociones. En los cuatro dominios de acción motriz, el procedimiento identificó correctamente como tales, las cuatro emociones positivas, las seis negativas y las tres ambiguas. Se reprodujo, por tanto, la estructura teórica de Bisquerra (2000, 2003) y Lazarus (2000) al clasificar las emociones en tres categorías en función de si se satisfacen o no las expectativas que se tiene al participar en un juego deportivo.

La validez convergente nos permitió asegurar que el GES se comportó de forma parecida al POMS aunque se hayan planteado desde marcos teóricos distintos. A pesar de que el POMS tiene un predominio de las emociones negativas, los 5 factores del POMS correlacionaron tal como se esperaba, con los 3 tipos de emociones del GES. El GES, al incorporar cuatro emociones positivas, permitió profundizar en esta clase de emociones, muy presentes en un gran mayoría de juegos deportivos.

El patrón de resultados obtenido permitió predecir las intensidades que cada emoción podía obtener en función del dominio de acción motriz. Aunque no se haya planteado como un análisis de validez predictiva, podemos afirmar que los juegos elicitarán diferentes intensidades de emociones en función de la situación que resulte de combinar dominio de acción motriz y resultado de la intervención. Esto coincide con las propiedades predictivas de los dominios de acción motriz, anunciadas por Parlebas (2001). En los juegos sin victoria, el escenario que plantea un juego psicomotor, donde el sujeto actúa solo, proporciona unas intensidades de emociones positivas ostensiblemente bajas. En cambio, al interactuar motrizmente con otros sujetos, esas intensidades son muy superiores, sobretodo en cooperación. En los juegos con victoria se generan dos escenarios distintos, ganar o perder, donde a su vez en función del dominio de acción motriz se generan intensidades muy distintas de emociones. Esta capacidad predictiva del GES al relacionar juegos deportivos y emociones teniendo en cuenta el resultado de la intervención, es de gran utilidad para el profesional de la actividad 
física y del deporte, en el momento de escoger el tipo de juego y las emociones que quiere provocar en función de su proyecto pedagógico o deportivo.

Por último, la validez de contenido confirmó que la estructura del GES es adecuada para estudiar las emociones que suscitan los juegos deportivos al aplicar el marco teórico de las emociones (Bisquerra, 2000) y de la praxiología motriz (Parlebas, 2001).

En cuanto a las limitaciones del estudio cabe indicar que el hecho que cuando el juego implica victoria y derrota, el sujeto no puede completar todos los ítems del cuestionario ya que si gana no puede expresar las emociones al perder, o al revés. Este hecho ha dificultado alguno de los análisis de validez llevados a cabo. También es necesario mencionar la cantidad de juegos utilizada en cada dominio de acción motriz. Será conveniente estudiar en futuras investigaciones la relación entre emociones y un mayor número de juegos por dominio.

\section{AGRADECIMIENTOS}

Este trabajo ha recibido financiación del Ministerio de Ciencia e Innovación. Proyectos I+D+i. Ref. DEP2010-21626-C03-01; DEP2010-21626-C03-02; DEP2010-21626-C03-03. También ha recibido financiación del Institut Català de les Dones (Generalitat de Cataluña). Ref. U-95/10. También ha recibido financiación de Agencia de Gestió d'Ajuts Universitaris i de Recerca de la Generalitat (AGAUR-INEFC). Ref. 2009SGR1404; VCP/3346/2009.

\section{REFERENCIAS}

Alfermann, D. (2003). Applications of diagnostic tools in exercise psychology. International Journal of Sport and Exercise Psychology, 1, 27-39. doi: 10.1080/1612197X.2003.9671702

Balaguer, I., Fuentes I., Melia J., García Merita M., \& Pérez Recio, G. (1994). El perfil de los estados de ánimo (POMS): Baremos para estudiantes valencianos y su aplicación al contexto deportivo. Revista de Psicología del deporte, 1, 35-52.

Bisquerra, R. (2000). Educación emocional y bienestar. Barcelona: Praxis.

Bisquerra, R. (2003). Educación emocional y competencias básicas para la vida. Revista de Investigación Educativa, 21 (1), 7-43.

Bredemeier, B. J. (1975). The assessment of reactive and instrumental athletic aggression. In D. M. Landers (Ed.), Psychology of sport and motor behaviour-II (pp.71-83). State Collegue, PA: Penn Satate HPER Series.

Collard, L., Oboeuf, A., \& Ahmaidi, S. (2007). The transfer of motor skills between swimming and gymnastics. Perceptual and motor skills, 105, 15-26.

Collard, L. (2008). Unfair sporting games and motor aggressiveness. Mathematics and Social Science, 182, 34-46.

Curran, P. J., West, S. G., \& Finch, J. F. (1996). The robustness of test statistics to nonnormality and specification error in confirmatory factor analysis. Psychological Methods, 1 (1), 16-29.

De Moya Martínez, M. V., Hernández Bravo, J. R., Hernández Bravo, J. A., Cózar Gutiérrez, R. (2011). Análisis de los estilos de aprendizaje y las TIC en la formación personal del alumnado universitario a través del cuestionario REATIC. Revista de Investigación Educativa, 29 (1), 137-156. 
Delaunay, M. (1981). Conduites sociomotrices, conflits et structures de jeu. Paris: INSEP.

Duda, J. L., Olson, L. K., \& Templin, T. J. (1991). The relationship of task and ego orientation to sportsmanship attitudes and the perceived legitimacy of injurious acts. Research Quarterly for Exercise and Sport, 62, 79-87.

Ekman, P., \& Davidson, R. J. (1994). The nature of emotion. Oxford, England: Oxford University Press.

García Calvo, T., Jiménez, R., Santos-Rsoa, F. J., Rena, R., \& Cervelló, E. (2008). Pscychometric properties of the spanish version of the flow state scale. The Spanish Journal of Pscychology, 2, 660-669.

Gauvin, L., \& Rejeski, W. J. (1993). The exercise-induced feeling inventory : Development and initial validation. Journal of Sport and Exercise Psychology, 15, 403-423.

Grove, J. R., \& Prapavessis, H. (1992). Preliminary evidence for the reliability and validity of an abbreviated Profile of Mood States. International Journal of Sport Psychology, 23, 93-109.

Hanin, Y. L. (Ed.) (2000). Emotions in sport. Champaign, IL: Human Kinetics.

Iglesias, M. J. (2009). Diagnóstico de las competencias emocionales: estudio empírico en la Facultad de Ciencias de la Educación de A Coruña (España). Revista de Investigación Educativa, 27 (2), 451-467.

Jackson, S. A., \& Marsh, H. W. (1996). Development and validation of a scale to measure optimal experience: The Flow State Scale. Journal of Sport and Exercise Psychology, 18 (1), 17-35.

Jackson, S. A., Kimiecik, J. C., Ford, S., \& Marsh, H. W. (1998). Psychological correlates of flow in sport. Journal of Sport and Exercise Psychology, 4, 358-378.

Jones, M. V., Lane, A. M., Bray, S. R., Uphill, M., \& Catlin, J. (2005). Development of the Sport Emotions Questionnaire. Journal of Sport and Exercise Psychology, 27, 407-431.

Jones, M. V., \& Sheffield, D. (2007). The impact of game outcome on the well-being of athletes. International Journal of Sport and Exercise Psychology, 5, 54-65.

Jones, G., Swain, A. B. J., \& Hardy, L. (1993). Intensity and direction dimensions of competitive state anxiety and relationships with performance. Journal of Sports Sciences, 1 (1), 525-532.

Kendzierski, D., \& DeCarlo, K. J. (1991). Physical Activity Enjoyment Scale: Two validation studies. Journal of Sport y Exercise Psychology, 13 (1), 50-64.

Lagardera, F. (1999). La lógica deportiva y las emociones. Implicaciones en la enseñanza del deporte. Revista Apunts de Educación Física, 56, 99-107.

Lane, A. M., Sewell, D. F., Terry, P. C., Bartram, D., \& Nesti, M. S. (1999). Confirmatory factor analysis of the Competitive State Anxiety Inventory 2. Journal of Sports Sciences, 17, 505-512.

Lane, A. M., \& Terry, P. C. (2000). The nature of mood: Development of a conceptual model with a focus on depression. Journal of Applied Sport Psychology, 12, 16-33.

Lazarus, R. S. (2000). How emotions influence performance in competitive sports. The Sport Psychologist, 14, 229-252.

Martens, R., Burton, D., Vealey, R. S., Bump, L. A., \& Smith, D. (1990). Development and validation of the Competitive State Anxiety Inventory.2. In R. Martens, R. S. Vealey, \& D. Burton (Eds.), Competitive anxiety in sport (pp. 117-190). Champaign, IL: Human Kinetics. 
McAuley, E., \& Courneya, K. (1994). The Subjective Exercise Experience Scale (SEES): Development and preliminary validation. Journal of Sport and Exercise Psychology, 16, 163-177.

McNair, D. M., Lorr, M., \& Droppleman, L. F. (1971). Profile of Mood State manual. San Diego, CA: Educational and Industrial Testing Service.

Morgan, W. P., Brown, D. R., Raglin, J. S., O'Connor, P. J., \& Ellickson, K. A. (1987). Psychological monitoring of overtraining and staleness. British Journal of Sports Medicine, 21, 107-114.

Mullen, R., Lane, A., \& Hanton, S. (2009). Anxiety symptom interpretation in highanxious, defensive high-anxious, low-anxious and repressor sport performers. Anxiety, Stress E Coping, 22 (1), 91-100.

Oboeuf, A., Collard, L., \& Gerard, B. (2008). Le jeu de la balle assise : un substitut au questionnaire sociométrique? [The game of the seated ball: a sociometric questionnaire substitute?]. Les Cahiers internationaux de psychologie sociale, 77, 87-100.

Parlebas P., \& Dugas E. (1998). Transfert d'apprentissage et domaines d'action motrice [Transfer of learning and motor action domains]. Education Physique et Sportive, 270, 41-47.

Parlebas, P. (2001). Juegos, deporte y sociedad. Léxico comentado de praxiología motriz. Barcelona: Paidotribo.

Smith, R. E., Smoll, F. L.,\& Schutz, R.W. (1990). Measurement and correlates of sportspecific cognitive and somatic trait anxiety: The Sport Anxiety Scale. Anxiety Research, 2, 263-280.

Terry, P. C., Lane, A. M., Lane, H. J., \& Keohane, L. (1999). Development and validation of a mood measure for adolescents: POMS-A. Journal of Sports Sciences, 17, 861-872.

Terry, P. C., Lane, A., \& Fogarty, G. (2003). Construct validity of the Profile of Mood States -Adolescents for use with adults. Psychology of Sport and Exercise, 4, 125-139. doi:10.1016/S1469-0292(01)00035-8

Uphill, M. A., \& Jones, M. V. (2007) Antecedents of emotions in elite athletes: a cognitive motivational relational theory perspective. Research Quaterly for Exercise and Sport. American Alliance for Health, Physical Education, Recreation and Dance, 78 (1), 79-89.

Watson, D., Clark, L., A., \& Tellegen, A. (1988). Development and validation of brief measures of positive and negative affect: The PANAS Scales. Journal of Personality and Social Psychology, 54, 1063-1070. doi:10.1037/0022-3514.54.6.1063

Zuckerman, M., \& Lubin, B. (1965). Manual for the Multiple Affect Adjective Checklist. San Diego, CA: Educational and Industrial Testing Service.

Fecha de recepción: 25 de febrero de 2012.

Fecha de revisión: 25 de febrero de 2012.

Fecha de aceptación: 23 de abril de 2012. 
ANEXO I.

Escala GES (Games and Emotion Scale)

Número Alumno/a:

Nombre del Juego:

Instrucciones: Lee atentamente la lista de emociones que se enumeran a continuación.

Después de leer cada emoción rodea con un círculo EL NÚMERO que mejor describa QUE SIENTES EN ESTE MOMENTO. Los números se ordenan según la intensidad manifestada: desde 0, que significa "nada", hasta 10, que se refiere a "muchísimo".

\begin{tabular}{|c|c|c|c|c|c|c|c|c|c|c|c|}
\hline $\begin{array}{l}\text { Resultado del } \\
\text { juego }\end{array}$ & \multicolumn{11}{|c|}{$\begin{array}{l}\text { Indica si el juego realizado es: } \\
\text { Juego sin Victoria } \square \\
\text { Juego con Victoria } \square \text { ¿Cómo has quedado en este juego? } \\
\text { Vencedor/a } \square \text { Perdedor/a } \square\end{array}$} \\
\hline FELICIDAD & 0 & 1 & 2 & 3 & 4 & 5 & 6 & 7 & 8 & 9 & 10 \\
\hline COMPASIÓN & 0 & 1 & 2 & 3 & 4 & 5 & 6 & 7 & 8 & 9 & 10 \\
\hline SORPRESA & 0 & 1 & 2 & 3 & 4 & 5 & 6 & 7 & 8 & 9 & 10 \\
\hline ALEGRÍA & 0 & 1 & 2 & 3 & 4 & 5 & 6 & 7 & 8 & 9 & 10 \\
\hline TRISTEZA & 0 & 1 & 2 & 3 & 4 & 5 & 6 & 7 & 8 & 9 & 10 \\
\hline MIEDO & 0 & 1 & 2 & 3 & & 5 & 6 & 7 & 8 & 9 & 10 \\
\hline HUMOR & 0 & 1 & 2 & 3 & & 5 & 6 & 7 & 8 & 9 & 10 \\
\hline ANSIEDAD & 0 & 1 & 2 & 3 & & 5 & 6 & 7 & 8 & 9 & 10 \\
\hline AMOR & 0 & 1 & 2 & 3 & & 5 & 6 & 7 & 8 & 9 & 10 \\
\hline IRA & 0 & 1 & 2 & 3 & & 5 & 6 & 7 & 8 & 9 & 10 \\
\hline RECHAZO & 0 & 1 & 2 & 3 & & 5 & 6 & 7 & 8 & 9 & 10 \\
\hline VERGÜENZA & 0 & 1 & 2 & 3 & & 5 & 6 & 7 & 8 & 9 & 10 \\
\hline ESPERANZA & 0 & 1 & 2 & 3 & 4 & 5 & 6 & 7 & 8 & 9 & 10 \\
\hline
\end{tabular}


